\title{
Labeling-Based Recipient Identification for 16-QAM BICM-ID
}

\author{
Maciej Krasicki (D)
}

\begin{abstract}
In recent years, many efforts have been made to find the optimal labeling maps for bit-interleaved coded modulation with iterative decoding (BICM-ID) with the aim to exploit the benefits of iterative decoding to the maximal extent. The current paper reveals new opportunities that BICM-ID signal labeling brings for system designers: it enables recipient addressing without any explicitly sent identifier. Instead, the recipient ID is represented by a labeling map, selected from a set of equally optimal labelings. A simple method of frame filtering, necessary to retrieve the desired data frame at the receiver side, is proposed and evaluated. It is also shown that the proposed Labeling-Based Recipient Identification (LABRID) approach does not cause any inferior performance outcome in terms of bit error rate.
\end{abstract}

Keywords: Early stopping, Iterative decoding, Modulation coding, TCP/IP, Turbo codes

\section{Introduction}

In a communication system, a message, to reach the final recipient, usually passes through several nodes and links, possibly over physical media of different types (twisted pair, wireless channel, etc.). To standardize the communication functions of the system without regard to its internal structure, the TCP/IP stack protocol [1] specifies four abstraction layers with some sublayers, like Medium Access Control (MAC). There is a specific protocol data unit (PDU) defined for each layer. When the transmission of PDU is ordered by a given layer, PDU is dropped to a lower layer and encapsulated in another PDU. As a consequence, the original message, generated in the highest layer is accompanied by a sizeable protocol overhead of each layer.

The physical layer is the one to provide a physical link for the TCP/IP's MAC protocols, together with protection from propagation errors. Thus, the data frame (i.e., PDU of MAC sublayer) is encapsulated in a so-called physical layer PDU (PPDU), whose structure depends on the coding scheme, modulation order, etc., for the given physical medium.

A wireless channel is shared by several stations. As a consequence, the data frame retrieved from the physical

Correspondence: maciej.krasicki@put.poznan.pl

Faculty of Electronics and Telecommunications, Chair of Wireless

Communications, Poznan University of Technology, Polanka 3, str., 61-131

Poznań, Poland layer at a given receiver might be actually destined for another station; such a foreign frame should be rejected. It is one of the reasons for which data frames contain a recipient identifier. In the case of a TCP/IP stack, the role of recipient identifier is played by the commonly known 48-bit MAC address (contrary to original assumptions, it is not worldwide unique anymore). At the receiver side, each PPDU must be decoded entirely, and shifted to MAC sublayer, where the data frame is de-encapsulated and its integrity is checked by means of CRC sum calculation. If the data frame passes the check, the recipient address is read and-based on it-the frame is determined as desired or foreign. All these steps must be done for either desired and foreign frames, due to the fact that the physical layer is "blind" to recipient addressing, which wastes receiver's energy. Additionally, the address field requires a sizeable amount of bandwidth resources to be passed through the channel, even more so as it undergoes channel coding, provided by the physical layer.

A future solution to avoid transmitting any recipient address field, thereby saving throughput and receiver power, might consist in reflecting the recipient identifier in some signal parameters. In the current paper, it is shown that-in the case of a BICM-ID system-it is enough to use different, carefully selected signal labeling maps, when transmitting the signal to particular users ${ }^{1}$, and the BICM-ID receiver can easily detect desired frames 
by considering the convergence of the iterative decoding process in the physical layer.

The paper just contributes the idea, and does not focus on changes in the TCP/IP protocol stack, necessary to enable dropping the recipient identifier to the physical layer instead of burying it into the data frame, etc. The paper structure is as follows. Section 2 briefly summarizes the essentials of BICM-ID. The proposed LABRID method is detailed in Section 3 and experimentally verified in Section 4. Finally, Section 5 concludes the work.

\section{BICM-ID essentials}

BICM-ID [2-5] is one of the most promising techniques for future wireless systems transmitting over Rayleigh fading channels. Similar to the serially concatenated turbo decoder [6], the BICM-ID receiver can improve decisions on data bits in subsequent decoding passes, where the demapper and soft-input soft-output convolutional decoder [7] exchange extrinsic information about codeword bits with one another. It is impossible for regular BICM, which has become a standard for the current wireless systems, due to the gray labeling map used therein. In recent years, BICM-ID had many variations, like bitinterleaved space-time coded modulation with iterative decoding [8], BICM-ID exploiting signal space diversity [9], bit-interleaved polar coded modulation with iterative decoding [10], or BICM-ID with multidimensional labeling $[11,12]$.

The iterative decoding process can converge if the following conditions are met: sufficient size of data frame, enough signal/noise ratio, and appropriate signal labeling. There is a so-called turbo cliff region on the BER vs. signal/noise ratio (SNR) plot, where experimental curves decline steeply and meet the so-called error-free feedback (EF) bound. The better the labeling map (or labeling, for brevity), the EF bound is shifted more and more downwards on the BER (SNR) plot. There have been many contributions devoted to find the optimal labeling, which provides the sharpest turbo cliff and the lowest position of the EF bound, e.g., $[13,14]$. The labeling "quality" is measured by the so-called asymptotic coding gain, i.e., the harmonic mean of Euclidean distance between constellation points, whose labels differ in exactly one bit position from each other:

$$
d_{E}^{2}(\omega)=\frac{1}{M 2^{M}}\left(\sum_{x \in \chi} \sum_{m=1}^{M} \frac{1}{\left|x-\bar{x}_{m}\right|^{2}}\right)^{-1},
$$

where $\bar{x}_{m}$ is the constellation point associated with the label differing only in bit position $m$ from the label assigned to $x$, according to labeling $\omega$. In this paper, the attention will be focused on $768 M 16^{a}$-compliant labelings found in [15]. Each of them exhibits exactly the same
$d_{E}^{2}=2.7145$, which is the second best value for BICM-ID (M16 ${ }^{r}$ is just slightly better) [8].

Along with the labeling, a channel code has an impact on the system performance, measured by bit error rate (BER). From the literature (e.g., [13]), it is known that short-memory codes result in early decoding convergence (i.e., the turbo cliff appears at low $E_{b} / N_{0}$ region), whereas long-memory codes give steeper EF bounds at the cost of shifting the turbo cliff towards the right on the BER vs. $E_{b} / N_{0}$ plot.

\section{LABRID and BICM-ID}

\subsection{Assumptions}

To explain Labeling-Based Recipient Identification (LABRID), let us start with a simple example. Imagine that you are leaving memo sticks for your family on the fridge door ("Bob, buy some butter, please"). Instead of writing their names on memo sticks, you use different pen colors, which correspond with different labelings in BICM-ID. The message ("buy some butter, please"), which is the counterpart of the data frame, is not touched, regardless of pen color; the same for the alphabet you use (it corresponds to the signal constellation), and the kind of memo sticks (the carrier). The only thing that identifies the message recipient is the color. Obviously, all your family members must recognize the "color code." It is similar to the case of BICM-ID, where each receiver must be able to reject the frames destined for the other ones.

LABRID emerges from the author's observation that the iterative decoding process does not converge at a given BICM-ID receiver if the received signal is labeled according to a labeling different than the one used at the current demapper. Therefore, it is proposed here to use 768 $M 16^{a}$-compliant labelings from [15] for recipient addressing. The choice of $M 16^{a}$ labeling and its replacements is justified by the following facts:

- High $d_{E}^{2}$ value simply makes them beneficial labelings for BICM-ID $[14,16]$ and also can help make a decision whether the iterative decoding process converges or not (the steep turbo cliff requires significant convergence of the iterative process).

- To the best of author's knowledge, it is the only known huge set of equivalent labelings. The use of equivalent labelings guarantees that none of the users is discriminated in a sense that the iterative decoding convergence is poorer than for any other user, which would result in different overall performance between the users.

By means of LABRID, one can address up to 768 receivers, which saves $\left\lceil\log _{2} 768\right\rceil=10$ bits under the assumption that the address in the reference solution is a simple number written in the natural binary code, without any protection against errors or protocol overhead. In a realistic 
scenario, where the recipient identifier role is played by the MAC address, protected by the channel code and CRC, the savings made with LABRID might be much more massive. Of course, LABRID does not pretend to be a globally unique addressing method. Nevertheless, it can cover a sufficient number of users within, e.g., a single LTE microcell or a WLAN.

\subsection{System model}

The block diagram of a BICM-ID system implementing LABRID is shown in Fig. 1. At the transmitter, data frame d is encoded by a [ 57$]_{8}$ convolutional encoder. (The simple $[57]_{8}$ encoder has been chosen with the aim to limit both simulation time and memory usage; a specific channel code is not a prerequisite for the LABRID method to be robust.) The codeword $\underline{\mathbf{u}}$ is passed through a random block bit-wise interleaver, having the interleaver depth equal to the codeword length. The interleaved codeword, $\underline{\mathbf{v}}$, is divided into binary blocks $\mathbf{v}_{1}, \ldots, \mathbf{v}_{K}$, each composed of $M=4$ bits. Every $\mathbf{v}_{k}$ block is mapped into a 16QAM signal $x_{k}=\omega_{i}\left(\mathbf{v}_{k}\right)$, according to a labeling map $\omega_{i}$, selected from the set containing all $768 M 16^{a}$-compliant labelings. In a real system, the recipient ID, $i$, is obtained as a result of cross-layer function execution. The signal is transmitted through an uncorrelated Rayleigh fading channel. The channel gain, $h_{k}$, is a complex Gaussian r.v. $\sim \mathcal{C N}(0,1)$ and the additive white Gaussian noise sample, $n_{k}$, is another complex Gaussian r.v. $\sim \mathcal{C N}\left(0, N_{0}\right)$, where $N_{0}$ stands for the noise power spectral density.

Let us consider all possible 768 receivers, each with its unique labeling applied to the demapper. Only one of the receivers (the $i$ th one) is the target recipient. With no generality loss, we assume that all the users acquire the same signal $y_{k}$, simultaneously.

The receivers operate according to the same principles as for the original BICM-ID: the demapper and SISO decoder, separated by bit-wise interleaver and deinterleaver, exchange their extrinsic information about codeword bits with each other. The extrinsic information (in the form of log-likelihood ratio (LLR)) is denoted by $\underline{\Lambda}^{[\mathrm{dem}, \mathrm{E}]}$ for the demapper and by $\underline{\boldsymbol{\Lambda}}^{[\mathrm{enc}, \mathrm{E}]}$ for the decoder. The demapper computes LLRs for each $k$ th binary block $\left(\mathbf{v}_{k}\right)$, separately, so that $\underline{\Lambda}^{[\mathrm{dem}, \mathrm{E}]}=\left[\boldsymbol{\Lambda}_{k}^{[\mathrm{dem}, \mathrm{E}]}\right]_{1 \leq k \leq K}$. The $\log$-MAP formula for the LLR related to the $m$ th bit of the $k$ th block is

$$
\Lambda_{k, m}^{[\mathrm{dem}, \mathrm{E}]}=\log \frac{\sum_{\boldsymbol{v}: v_{m}=0} \exp \left(\Gamma_{k, \boldsymbol{v}}+\boldsymbol{\Lambda}_{k}^{[\mathrm{dem}, \mathrm{A}]} \boldsymbol{v}^{T}\right)}{\sum_{\boldsymbol{v}: v_{m}=1} \exp \left(\Gamma_{k, \boldsymbol{v}}+\boldsymbol{\Lambda}_{k}^{[\mathrm{dem}, \mathrm{A}]} \boldsymbol{v}^{T}\right)}-\Lambda_{k, m}^{[\mathrm{dem}, \mathrm{A}]}
$$

where $\Gamma_{k, \boldsymbol{v}}=\log \frac{1}{\pi N_{0}}-\frac{1}{N_{0}}\left|y_{k}-h_{k} \omega_{j}(\boldsymbol{v})\right|^{2}$ is the information about the transmitted signal, acquired from the channel. In LABRID, each receiver employs its unique

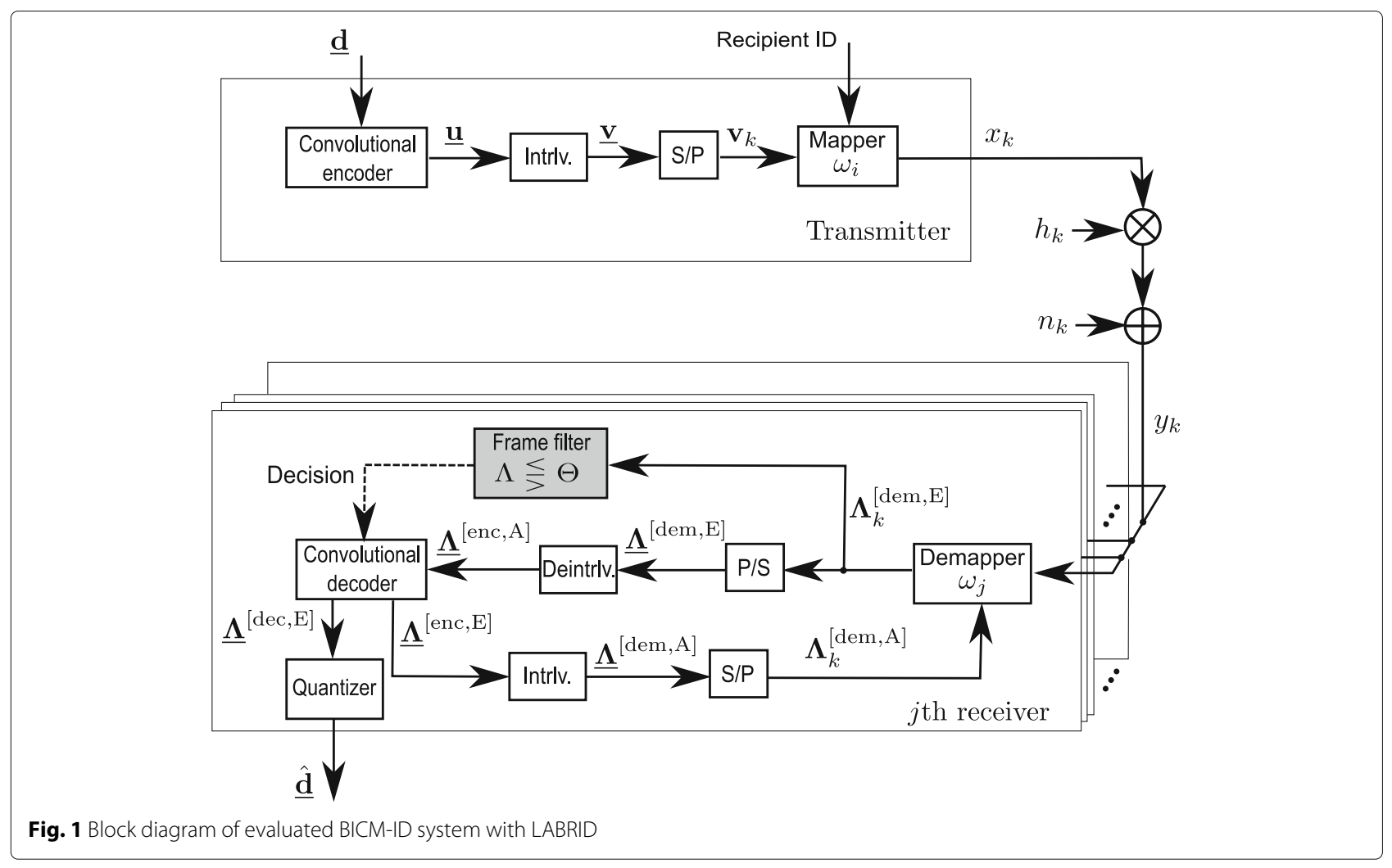


labeling map, $\omega_{j}$, so that $\Gamma_{k, v}$ depends on the current receiver ID, $j$. More specifically, $\omega_{j}(\boldsymbol{v})$ is a $16-\mathrm{QAM}$ signal associated with a hypothetically considered $M$-bit-long block $\boldsymbol{v}$, according to labeling $\omega_{j}$. Finally, $\boldsymbol{\Lambda}_{k}^{[\mathrm{dem}, \mathrm{A}]}$ is the a priori demapper LLR vector related to the binary block $\mathbf{v}_{k}$, obtained from the SISO decoder. The decoder employs the log-ML algorithm, presented in [7].

Along with the pure BICM-ID receiver structure, described above, there is a block (detailed in the next section) responsible for frame filtering. If the decision of the block is to treat the current data frame as the desired data frame, the SISO decoder computes a vector of LLRs, related to the data bits (the vector is denoted by $\underline{\Lambda}^{[\mathrm{dec}, \mathrm{E}]}$ ). Passing this vector through a two-level quantizer gives final binary decisions, $\hat{\mathbf{d}}$, on the data bits.

\subsection{Frame filtering}

If LABRID is to be robust, each receiver must be able to correctly detect desired frames and discard those which are not destined for it (foreign frames). Thus, the LABRID-compatible receiver needs a method for frame filtering. Actually, the goal of frame filtering is to discard the frames for which the iterative process does not converge. It must be pointed out that the lack of decoding convergence can suggest not only a foreign frame, but also a desired frame which cannot be decoded correctly, due to, e.g., poor channel conditions. However, such ambiguity does not cause any deterioration of the system performance, since any desired-but-undecodable frame is useless for either LABRID-compatible or regular BICM-ID receiver.

Frame filtering might rely on the Cyclic Redundancy Check method, commonly used in wireless systems to verify data frame integrity. Herein, we suggest another solution, which does not require any redundant bits to be attached to the data frame. The method is based on a feature of the BICM-ID system that can predict both data estimates' accuracy and BER given extrinsic demapper LLRs [17]. It is possible thanks to the extrinsic information transfer curves, which characterize both the BICMID demapper and decoder, and are commonly used for EXIT analysis of iteratively decoded systems [18]. Another important fact is that the mutual information between extrinsic demapper LLRs $\underline{\Lambda}^{[\mathrm{dem}, \mathrm{E}]}$ and the codeword $\underline{\mathbf{v}}$ is reflected in the absolute mean of the former, denoted by $\left\langle\left|\underline{\Lambda}^{[\mathrm{dem}, \mathrm{E}]}\right|\right\rangle$. This connection is exploited in an early stopping method for turbo codes, called Mean Estimate [19]. Thus, frame filtering consists in comparing $\left\langle\left|\underline{\Lambda}^{[\mathrm{dem}, \mathrm{E}]}\right|\right\rangle$ with an empirical threshold $\Theta$. If $\left\langle\left|\underline{\Lambda}^{[\mathrm{dem}, \mathrm{E}]}\right|\right\rangle$ increases through subsequent iterations and finally exceeds the threshold, the frame is considered a desired frame. Otherwise, the frame is rejected as foreign.
To sum up, there is no need to obtain the data estimates for frame filtering purposes, and the decision about the frame destination (desired or foreign) can be made basing on a simple threshold criterion. Another advantage of the threshold-based method over the CRC-aided method is that the former does not require any redundant bits.

\subsection{Analysis of incorrect decision probability}

There is a risk that the decision on the frame destination is incorrect. In fact, there are two kinds of errors:

- Misdetection, when a desired decodable frame is rejected.

- False alarm, when a foreign frame is believed to be a desired frame.

Note that a frame is truly desired by the $j$ th receiver, if the signal has been labeled according to $\omega_{j}$ at the transmitter. For any $\omega_{i \neq j}$ labeling used at the transmitter, the frame is truly foreign. Let us treat the observed metric, $\left\langle\left|\underline{\Lambda}^{[\mathrm{dem}, \mathrm{E}]}\right|\right\rangle$, computed by the $j$ th receiver as a random variable $\Lambda$. The misdetection event appears for the truly desired decodable frame if the observed metric $\Lambda$ is below the threshold $\Theta$. Thus, the misdetection ratio (MR) for the $j$ th receiver is

$$
\operatorname{MR}_{j}(\Theta)=\int_{0}^{\Theta} p_{\Lambda \mid \omega_{j}}\left(\lambda \mid \omega_{j}\right) \mathrm{d} \lambda .
$$

where $p_{\Lambda \mid \omega_{j}}$ is the probability density function (PDF) of $\Lambda$ given the fact that the frame is truly desired, i.e., the transmitter uses labeling $\omega_{j}$.

If the transmitter uses labeling $\omega_{i \neq j}$ and metric $\Lambda$ observed at receiver $j$ is above threshold $\Theta$, a false alarm event appears. So, given particular $\omega_{i}$, the false alarm rate (FAR) at receiver $j$ reads

$$
\operatorname{FAR}_{j, i}(\Theta)=\int_{\Theta}^{\infty} p_{\Lambda \mid \omega_{i \neq j}}\left(\lambda \mid \omega_{i \neq j}\right) \mathrm{d} \lambda .
$$

The total erroneous decision probability at receiver $j$ depends on the data traffic characteristics, i.e., the number of data frames directed to different destinations, which is reflected in the probability of using specific labelings at the transmitter:

$$
P_{j}^{E}(\Theta)=\mathrm{MR}_{j}(\Theta) \cdot P\left(\omega_{j}\right)+\sum_{i \neq j} \mathrm{FAR}_{j, i}(\Theta) \cdot P\left(\omega_{i}\right) .
$$

Since all the $M 16^{a}$-compliant labelings have exactly the same features, $\mathrm{FAR}_{j, i}$ values are the same for each $(i, j \neq i)$ pair. Similarly, MR does not depend on the considered labeling $\omega_{j}$. As a consequence, we can write

$$
P_{j}^{E}(\Theta)=\operatorname{MR}(\Theta) \cdot P\left(\omega_{j}\right)+\operatorname{FAR}(\Theta) \sum_{i \neq j} P\left(\omega_{i}\right) .
$$


In order to simplify our considerations, let us assume that the signal is directed to each of 768 receivers with the same probability; we arrive at

$$
P^{E}(\Theta)=\frac{1}{768} \mathrm{MR}(\Theta)+\frac{767}{768} \mathrm{FAR}(\Theta),
$$

valid for each receiver. It must be kept in mind that both conditional PDFs $p_{\Lambda \mid \omega_{j}}$ and $p_{\Lambda \mid \omega_{i \neq j}}$ might depend on the current $E_{b} / N_{0}$ ratio. In consequence, the optimal threshold,

$$
\Theta_{\mathrm{opt}}=\arg \min _{\Theta \in[0, \infty)} P^{E}(\Theta),
$$

should be considered separately for each $E_{b} / N_{0}$ setting. The second observation is that $P^{E}$ might be dominated by FAR, in particular when the number of system users is very high.

\subsection{Compatibility consideration}

The proposed LABRID is fully transparent for the older devices in a BICM-ID system, which use MAC addresses, transmitted explicitly as a separate data frame field. Let us assume that the labeling used by all legacy BICM-ID devices is the original $M 16^{a}$ labeling from [14]. With no generality loss, let us name it $\omega_{1}$. During transmission in the legacy mode (between older devices), the original $\omega_{1}$ labeling is used each time, and the recipient is identified by the appropriate recipient address field in the data frame. Meanwhile, in the greenfield mode, applicable when transmitting between two LABRID-compatible devices, the transmitter applies an appropriate recipientcharacteristic labeling map, other than $\omega_{1}$. The signals transmitted in the greenfield mode would be discarded by the older devices unable to retrieve any valid addresses from the undecodable data frame (possibly followed by an incorrect CRC sum).

LABRID does not interfere with the ARQ (Automatic Repeat reQuest) mechanisms, implemented in telecommunication systems to confirm the reception of subsequent data frames. As a part of the ARQ procedure, an acknowledge (ACK) frame is transmitted from the message recipient to the sender. For better illustration, let us take into account the 802.11-family WLAN specifications. According to them, the ACK frame is nothing more than a regular data frame (i.e., PDU of MAC sublayer) with some control bits set. If LABRID is to be used, the ACK frame is dropped down to the physical layer along with the recipient ID (this time, it is the identifier of the original message sender), and the physical layer assigns appropriate labeling, as usually.

\subsection{LABRID with modulations different than 16-QAM}

Most of the wireless systems implement a rate matching mechanism with the aim of maximizing the throughput, while preserving a reasonable bit error rate. It might consist in dynamic modulation order switching. Formally, there is no impediment that limits the application of LABRID to systems with 16-QAM modulation only. The problem is that-as for now-there is no algorithm to determine a complete set of equivalent labelings for highorder modulations (like 64-QAM or 256-QAM) in just a few seconds. However, some of them can be found in a reasonable time by means of general-purpose optimization algorithms (binary switching algorithm [14], reactive tabu search (RTS) [20], etc.), used in the past to search for the optimal BICM-ID labelings $[8,13,14]$. This is not just a theoretic statement, as the author of this paper succeeded in finding 24 labelings equivalent to the optimal 64-QAM one from [8], which exhibits $d_{E}^{2}=2.8742$. Therefore, it is only a matter of time to get the desired set of equivalent labelings.

In terms of low-order modulations, like QPSK and BPSK, they do not offer significant convergence of iterative decoding, and there is even not much choice of labelings due to the limited number of constellation points. For this reason, these modulations are poor candidates for either LABRID or even pure BICM-ID. However, the decoding convergence of such low-order modulations can be substantially improved by means of multidimensional (hypercube) labelings, as reported in [21]. Thus, LABRID seems to be applicable for BICM-ID with BPSK, QPSK, or 8-PSK, unless there are no sets of equivalent multidimensional labelings. This matter requires further study and is not covered herein.

\section{Simulation results and discussion}

A simulation model has been developed according to the description in Section 3.2. At first, BER vs. $E_{b} / N_{0}$ is measured at the destined receiver to check if the use of replacement labelings impacts the system performance to any extent. Additionally, the frame size, necessary to obtain the effect of decoding convergence, is assessed. Afterwards, the proposed threshold-based method for frame filtering is investigated.

\subsection{BER vs. $E_{b} / N_{0}$ performance}

Let us assume that the transmitter labeling map, $\omega_{i}$, is generated randomly from 768 candidate $M 16^{a}$-compliant labelings, and we observe the number of erroneously decided bits at the destined receiver, i.e., the one using $\omega_{j=i}$ labeling map. We compare the result with the theoretical EF bound for the original $M 16^{a}$-labeled BICM-ID [14], computed according to the method presented in $[22,23]$. The considered frame size is either 200 or 1000 bits. The result, observed in selected iterations, is shown in Fig. 2. In the left chart, one can see an insignificant BER drop in iterations 2 through 25, which is a sign of poor decoding convergence. Such a 

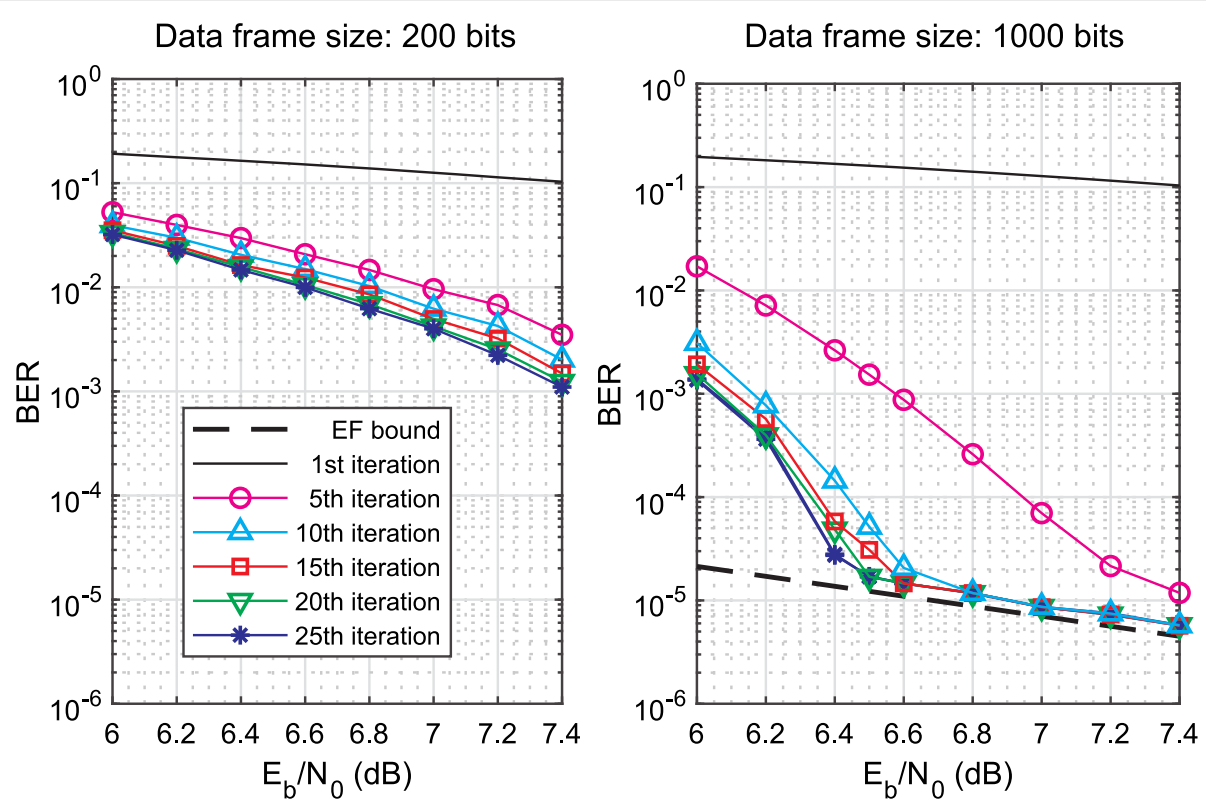

Fig. 2 BER vs. $E_{b} / N_{0}$ for different data frame sizes

drawback is not LABRID-specific-it can also be observed for the original BICM-ID. (If the frame is short, there is no chance to spread subsequent codeword bits to such extent, which allows considering the entries of $\boldsymbol{\Lambda}_{k}^{[\mathrm{dem}, \mathrm{A}]}$ to be independent from each other and then the iterative process stucks.) For the frame size of 1000 bits (the right plot), the desired turbo cliff appears at $E_{b} / N_{0}$ range between 6 and $6.5 \mathrm{~dB}$, and for higher $E_{b} / N_{0}$, the simulation curves follow the EF bound. This means that the frame size of 1000 bits is enough for the iterative decoding to converge. The fact that the simulation curves reach the analytical EF bound of $M 16^{a}$-labeled BICM-ID shows that LABRID itself does not cause any performance gap.

\subsection{Frame filtering examination}

The crucial part of LABRID is a mechanism for discarding foreign frames. In Section 3.3, it was suggested that it is enough to check if the absolute mean of extrinsic demapper LLR for a given frame, $\left\langle\left|\underline{\Lambda}^{[\mathrm{dem}, \mathrm{E}]}\right|\right\rangle$, exceeds a pre-assummed threshold $\Theta$. For the method to be robust, the $\left\langle\left|\underline{\Lambda}^{[\mathrm{dem}, \mathrm{E}]}\right|\right\rangle$ value must be significantly different for foreign and desired frames. To show if this is the case, we define the following simulation setup. For each data frame, first, the receiver's labeling map $\omega_{j}$ is selected randomly, and then two cases of the transmitter's labeling are considered:

- $\omega_{i=j}$ (it means that the receiver acquires a desired frame)
- Randomly selected $\omega_{i \neq j}$ (the receiver gets a foreign frame, which should be rejected)

Given the labelings, we empirically determine conditional PDFs $p_{\Lambda \mid \omega_{j}}$ and $p_{\Lambda \mid \omega_{i \neq j}}$ in subsequent iterations and average them over the transmitted data frames. Figure $3 \mathrm{a}$ and $\mathrm{b}$ show the results for $E_{b} / N_{0}$ equal to $6 \mathrm{~dB}$ and $7.4 \mathrm{~dB}$, respectively. For each setup, the transmission of $10^{6}$ data frames, each counting 1000 bits, is simulated. The probability below $10^{-5}$ is not shown for the reason that very rare PDF entries might be statistically unreliable [24].

In both figures, it can be seen that the histogram peak for the foreign frames does not evolve from one iteration to another, and the median is slightly higher for $E_{b} / N_{0}=$ $7.4 \mathrm{~dB}$ than for $6 \mathrm{~dB}$. Meanwhile, for the desired frames, the PDF in the first iteration is almost the same as for foreign frames, but its peak significantly moves towards higher entries, which is a clear sign of decoding convergence. However, at $E_{b} / N_{0}=6 \mathrm{~dB}$, there are still some entries below $\lambda=10$, which complicates setting the threshold. There are no such cases at $E_{b} / N_{0}=7.4 \mathrm{~dB}$ and the PDFs for desired and foreign frames diverge after a few iterations; it is proof that the proposed frame filtering method is robust.

Based on the conditional PDFs $p_{\Lambda \mid \omega_{j}}$ and $p_{\Lambda \mid \omega_{i \neq j}}$, MR and FAR are calculated in the 25th iteration for different values of threshold $\Theta$ and $E_{b} / N_{0}$ and drawn on a common plot (Fig. 4). Again, the results below $10^{-5}$ are not displayed, since they might be statistically unreliable. As a consequence, we cannot search for the optimal $\Theta_{\text {opt }}$ directly, based on the numbers shown in the figure. 


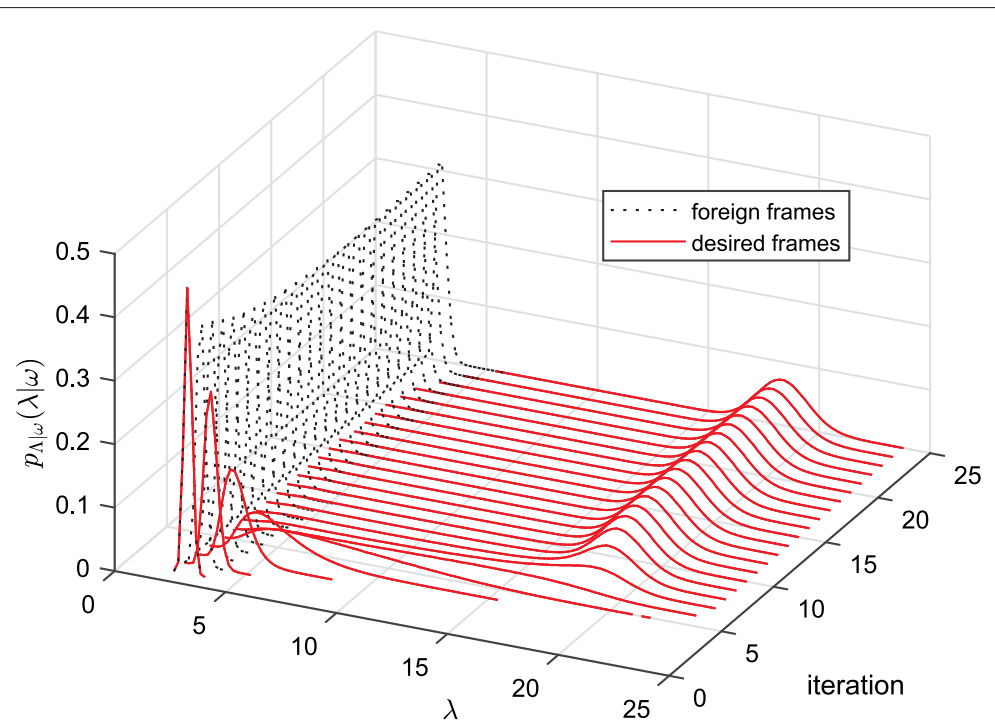

(a)

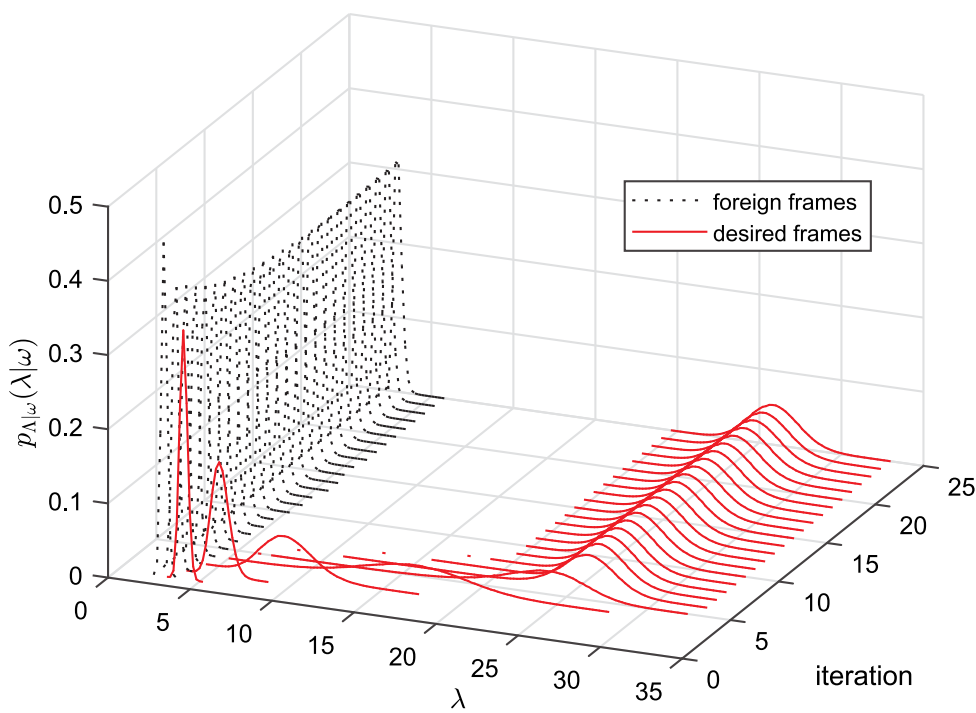

(b)

Fig. 3 Empirical probability of $\left\langle\left|\underline{\boldsymbol{\Lambda}}^{[\mathrm{dem}, \mathrm{E}]}\right|\right\rangle$ through consecutive iterations at $E_{b} / N_{0}=6 \mathrm{~dB}(\mathbf{a})$ and $7.4 \mathrm{~dB}(\mathbf{b})$

Instead, let us set a provisional optimization goal: $\mathrm{MR} \leq$ $10^{-5}$ and FAR $\leq 10^{-5}$ at the same time. If we manage to find a threshold for which both criteria are met, we get $P^{E} \leq 10^{-5}$ according to Eq. 7 .

As can be seen from Fig. 4, the FAR surface has a very sharp edge. It results from a very low dispersion of $p_{\Lambda \mid \omega_{i \neq j}}$, i.e., a small increment in the considered threshold value in the range $(2.5,5)$ significantly increases the area under the respective $p_{\Lambda \mid \omega_{i \neq j}}$ curve in Fig. 3b, which, simply, constitutes FAR according to Eq. 4. The FAR dependence on $E_{b} / N_{0}$ is very weak: FAR at the level of $10^{-5}$ is reached for $\Theta=5.3$ at $E_{b} / N_{0}=6 \mathrm{~dB}$ and increases slightly, up to
5.7 at $E_{b} / N_{0}=7.4 \mathrm{~dB}$. Thus, to avoid a FAR bigger than $10^{-5}$, it is enough to set $\Theta>5.7$, regardless of the current $E_{b} / N_{0}$.

The optimal threshold value is, additionally, upperbounded due to MR requirements. The MR surface falls down sharply, as the FAR surface does, but for lower $E_{b} / N_{0}$ (in the range between $6 \mathrm{~dB}$ and $c a .6 .6 \mathrm{~dB}$ ), a significant lobe appears. Thus, the threshold should be adjusted carefully to match the narrow gap between MR and FAR surfaces at $E_{b} / N_{0}=6 \mathrm{~dB}$. However, from the BER vs. $E_{b} / N_{0}$ plot (Fig. 2), it can be seen that BER is still unreasonably high at $E_{b} / N_{0}=6 \mathrm{~dB}\left(c a \cdot 10^{-3}\right)$, and hence, it 


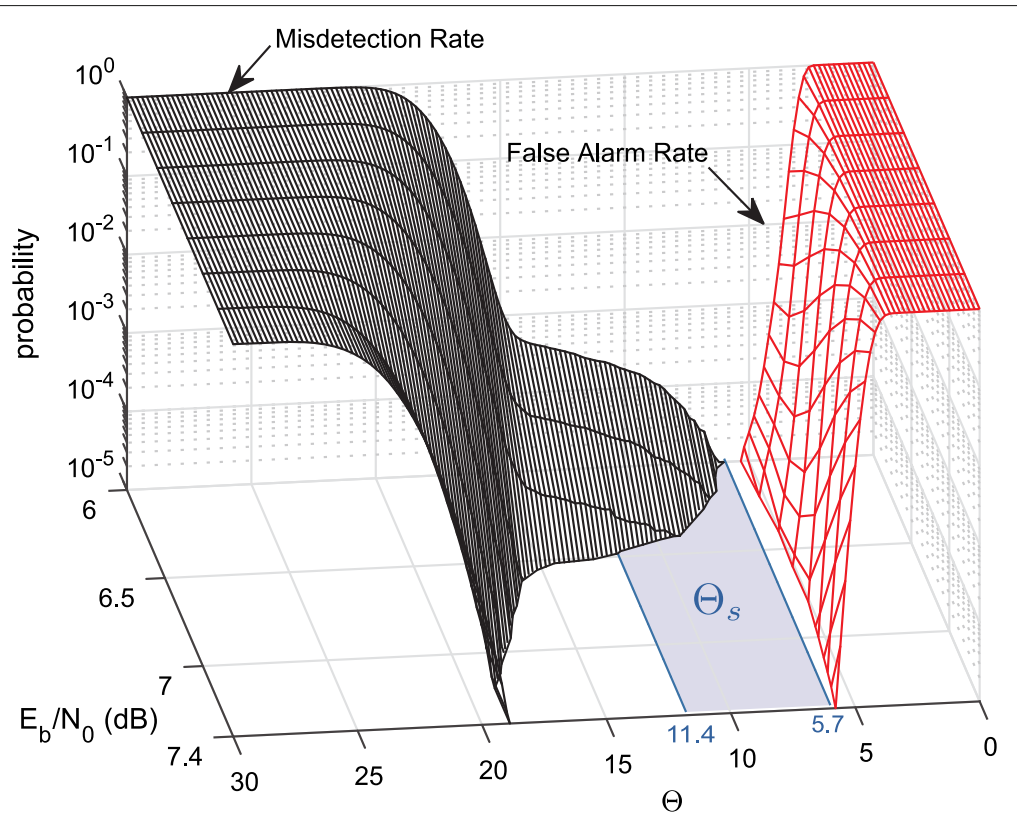

Fig. 4 False alarm rate and misdetection rate vs. $E_{b} / N_{0}$ vs. threshold level

might be out of interest. In fact, BER curves reach their EF bound at $E_{b} / N_{0} \approx 6.5 \mathrm{~dB}$, where the lobe from Fig. 4 has already started to recede. Thus, to avoid refining the SNRdependant $\Theta_{\text {opt }}$, the author suggests setting a constant threshold value, $\Theta_{s}$. It can be any number between 5.7 and 11.4. As can be seen from Fig. 4, a strip representing the suggested range crosses the MR lobe at $E_{b} / N_{0}=6.5 \mathrm{~dB}$ (where BER becomes unacceptably high) and does not cross the FAR slope at all ${ }^{2}$.

In the current experiment, the frame filter makes its decision in the last executed (25th) iteration. In fact, the number of iterations to be passed before the frame destination is judged seems to be overestimated, as the $p_{\Lambda \mid \omega_{j}}$ histograms from Fig. 3b do not evolve for the 9th and subsequent iterations at $E_{b} / N_{0}=7.4 \mathrm{~dB}$. It suggests reducing the number of considered iterations. But from the BER plots (Fig. 2), it might be seen that in the 10th iteration the system performance for $E_{b} / N_{0}$ below $6.8 \mathrm{~dB}$ is slightly deteriorated in comparison with the result observed in the 25th iteration (compare the lines with $\triangle$ and $*$ markers). As a future solution to the problem of overestimated number of iterations, frame filtering might be incorporated into the original ME early-stopping method so that every frame qualified as error-free is considered to be a desired decodable frame at the same time. Thanks to that, subsequent frames would be decoded in a different number of (exactly required) iterations, thus reducing the average.

\section{Conclusions}

The proposed recipient identification method in BICMID, called LABRID, consists in using recipient-specific labelings, which belong to a set of equally good $M 16^{a}$ compliant labelings. It does not waste any bit for sending recipient address within the data frame. Another benefit of LABRID is that foreign frames can be rejected early on in the physical layer by means of a simple threshold-based frame filtering procedure. The only costs of the LABRIDcompatible equipment over conventional BICM-ID are the frame filter and the mechanism to set appropriate labeling at the mapper before sending a given frame (a simple look-up table would be enough). The reference labeling at the receiver side must be set only once-during the initialization phase.

The question left for the future research is if LABRID could be adapted to modulations different than 16-QAM. It would imply more studies on sets of equivalent labelings. Another issue that requires future analysis is the proposal to merge the process of frame filtering with an early-stopping mechanism, suggested at the end of Section 4.2.

\section{Methods/Experimental}

To obtain the results presented in the paper, a simulation experiment was conducted on a PC machine. All of the MATLAB code has been developed by the author, except for built-in functions. All data bits as well as the channel states were generated randomly. The simulation was conducted according to the Monte Carlo principles.

\section{Endnotes}

${ }^{1}$ Note the difference between a label and a labeling map (or, simply, labeling) - the former is a binary sequence 
associated with given signal parameters (like phase and/or amplitude) according to rules described by the latter.

${ }^{2}$ The fact that the right strip edge meets the MR lobe at $E_{b} / N_{0}=6 \mathrm{~dB}$ has no practical implication.

\section{Abbreviations}

BER: Bit-error rate; BICM-ID: Bit-interleaved coded modulation with iterative decoding; EF: Error-free feedback; EXIT: Extrinsic-information transfer; FAR: False alarm ratio; LABRID: Labeling-Based Recipient Identification; LLR: Log-likelihood ratio; MAC: Medium Access Control; MR: Misdetection ratio; PDF: Probability density function; PDU: Protocol data unit; PPDU: Physical layer PDU; SNR: Signal/noise ratio

\section{Acknowledgements}

Not applicable.

\section{Authors' contributions}

MK has invented LABRID, conducted simulation experiments, and is the only author of the paper. The author read and approved the final manuscript.

\section{Funding}

The presented work has been funded by the Polish Ministry of Science and Higher Education.

\section{Availability of data and materials}

The data presented in the manuscript can be obtained easily by performing MATLAB programming according to the formulas provided in this manuscript. There is no need to generate the datasets for storage.

\section{Competing interests}

The author declares that he has no competing interests.

Received: 12 March 2019 Accepted: 24 June 2019

Published online: 09 July 2019

\section{References}

1. IETF: RFC1180: A TCP/IP Tutorial (IETF, 1991). https://tools.ietf.org/html/ rfC 1180

2. X. Li, J. A. Ritcey, Bit-interleaved coded modulation with iterative decoding. 1(6), 169-171 (1997). https://doi.org/10.1109/4234.649929

3. X. Li, J. A. Ritcey, in IEEE International Conference on Communications (ICC '99). Bit-interleaved coded modulation with iterative decoding, vol. 2, (1999), pp. 858-863. https://doi.org/10.1109/ICC.1999.765396

4. J. Tan, G. L. Stuber, Analysis and design of symbol mappers for iteratively decoded BICM. 4(2), 662-672 (2005). https://doi.org/10.1109/TWC.2004. 842966

5. N. H. Tran, H. H. Nguyen, in Canadian Conference on Electrical and Computer Engineering. Signal mappings of 8-ary constellations for BICM-ID systems over a Rayleigh fading channel, vol. 3, (2004), pp. 1809-1813. https://doi.org/10.1109/CCECE.2004.1349768

6. S. Benedetto, G. Montorsi, Iterative decoding of serially concatenated convolutional codes. Electron. Lett. 32(13), 1186-1188 (1996). https://doi. org/10.1049/el:19960793

7. S. Benedetto, D. Divsalar, G. Montorsi, F. Pollara, A soft-input soft-output APP module for iterative decoding of concatenated codes. 1(1), 22-24 (1997). https://doi.org/10.1109/4234.552145

8. Y. Huang, J. A. Ritcey, Optimal constellation labeling for iteratively decoded bit-interleaved space-time coded modulation. 51(5), 1865-1871 (2005). https://doi.org/10.1109/TIT.2005.846409

9. Q. Xie, K. Peng, F. Yang, Z. Wang, in 2010 IEEE Global Telecommunications Conference GLOBECOM 2010. BICM-ID systems with signal space diversity over Rayleigh fading channels, (2010), pp. 1-5. https://doi.org/10.1109/ GLOCOM.2010.5683457

10. S. Saha, M. Tschauner, M. Adrat, T. Schmitz, P. Jax, P. Vary, in 2018 International Conference on Military Communications and Information Systems (ICMCIS). Bit-interleaved polar coded modulation with iterative decoding, (2018), pp. 1-8. https://doi.org/10.1109/ICMCIS.2018.8398698

11. H. M. Navazi, M. J. Hossain, Efficient multi-dimensional mapping using QAM constellations for BICM-ID. IEEE Transactions on Wireless
Communications. 16, 8067-8076 (2017). https://doi.org/10.1109/twc. 2017.2756837

12. H. M. Navazi, M. J. Hossain, Novel method for multi-dimensional mapping of higher order modulations for BICM-ID over Rayleigh fading channels. IEEE Transactions on Wireless Communications. 18(2), 1142-1154 (2019). https://doi.org/10.1109/TWC.2018.2890061

13. A. Chindapol, J. A. Ritcey, Design, analysis, and performance evaluation for BICM-ID with square QAM constellations in Rayleigh fading channels. 19(5), 944-957 (2001). https://doi.org/10.1109/49.924878

14. F. Schreckenbach, N. Gortz, J. Hagenauer, G. Bauch, in IEEE Global Telecommunications Conference (GLOBECOM '03). Optimized symbol mappings for bit-interleaved coded modulation with iterative decoding, vol. 6, (2003), pp. 3316-3320. https://doi.org/10.1109/GLOCOM.2003. 1258849

15. M. Krasicki, Algorithm for generating all optimal 16-QAM BI-STCM-ID labelings. Wirel. Pers. Commun. 83, 873-894 (2015). https://doi.org/10. 1007/s11277-015-2431-1

16. F. Schreckenbach, N. Gortz, J. Hagenauer, G. Bauch, Optimization of symbol mappings for bit-interleaved coded modulation with iterative decoding. 7(12), 593-595 (2003). https://doi.org/10.1109/LCOMM.2003. 821325

17. T. Clevorn, S. Godtmann, P. Vary, BER prediction using EXIT charts for BICM with iterative decoding. 10(1), 49-51 (2006). https://doi.org/10.1109/ Icomm.2006.1576566

18. J. Hagenauer, in Proc. 12th Europ. Signal Processing Conference (EUSIPCO). The EXIT chart - introduction to extrinsic information transfer (Institute of Communications and Radio-Frequency Engineering, Vienna, 2004), pp. 1541-1548

19. F. Zhai, I. J. Fair, Techniques for early stopping and error detection in turbo decoding. IEEE Trans. Commun. 51(10), 1617-1623 (2003). https://doi. org/10.1109/TCOMM.2003.818099

20. R. Battiti, G. Tecchiolli, The Reactive Tabu Search. ORSA Journal on Computing. 6(2), 126-140 (1994). https://doi.org/10.1287/ijoc.6.2.126. http://arxiv.org/abs/http://dx.doi.org/10.1287/ijoc.6.2.126

21. N. H. Tran, H. H. Nguyen, in IEEE 60th Vehicular Technology Conference, 2004. VTC2004-Fall. 2004. Improving the performance of QPSK BICM-ID by mapping on the hypercube, vol. 2, (2004), pp. 1299-13032. https://doi. org/10.1109/NETECF.2004.1400233

22. S. Benedetto, E. Biglieri. Principles of digital transmission with wireless applications (Kluwer Academic Publishers, New York, 1999), pp. 702-708

23. E. Biglieri, G. Caire, G. Taricco, J. Ventura-Traveset, Simple method for evaluating error probabilities. Electron. Lett. 32(3), 191-192 (1996). https://doi.org/10.1049/el:19960148

24. M. C. Jeruchim, P. Balaban, K. S. Shanmugan. 2nd edn., in Simulation of Communication Systems. Error sources in simulation (Plenum Press, New York, 2002), pp. 622-629

\section{Publisher's Note}

Springer Nature remains neutral with regard to jurisdictional claims in published maps and institutional affiliations.

\section{Submit your manuscript to a SpringerOpen ${ }^{\odot}$ journal and benefit from:}

- Convenient online submission

- Rigorous peer review

- Open access: articles freely available online

- High visibility within the field

- Retaining the copyright to your article

Submit your next manuscript at $>$ springeropen.com 